\title{
Endoscopic-assisted Craniofacial Resection
}

\author{
${ }^{1}$ Prathamesh S Pai, ${ }^{2}$ Alias gar Moiyadi, ${ }^{3}$ Deepa Nair \\ ${ }^{1}$ Associate Professor, Department of Head and Neck Surgical Oncology, Tata Memorial Centre, Mumbai, Maharashtra, India \\ ${ }^{2}$ Associate Professor, Department of Neurosurgery, Tata Memorial Centre, Mumbai, Maharashtra, India \\ ${ }^{3}$ Assistant Professor, Department of Head and Neck Surgical Oncology, Tata Memorial Centre, Mumbai, Maharashtra, India
}

Correspondence: Prathamesh S Pai, Associate Professor, Department of Head and Neck Surgical Oncology Tata Memorial Hospital, Parel, Mumbai 400012, Maharashtra, India, Phone: +91 22 24177185, Fax: +91 2224146937 e-mail: drpspai@gmail.com

\section{ABSTRACT}

Management of anterior skull base tumors has progressed steadily since AS Ketcham popularized the craniofacial surgical technique in the seventies with good results. In the past two decades, endoscopic sinonasal tumor resection has been established as an additional treatment option. For tumors that cross the anterior skull base, a cranial access is vital to encompass the tumor all around. For a select group of these transcranial lesions, the sinonasal component is suitable for an endoscopic endonasal oncologically safe resection along with a traditional transcranial access to complete the resection. This article endeavors to describe the endoscopicassisted craniofacial combining the advantages of a transnasal minimal access to reduce facial morbidity and the transcranial access for superior control of tumors with adequate margins.

Keywords: Paranasal sinus neoplasms, Endoscopy, Craniofacial resection.

\section{INTRODUCTION}

The anterior skull base region extends from the frontal sinus anteriorly to the clivus inferiorly in the sagittal plane and the region between the orbits in the coronal plane. Tumors in this region can arise from a wide variety of structuressinonasal mucosa, skull bone, olfactory fibers, meninges, orbit, pituitary, optic nerve and the vessels. These tumors are rare and often tend to present dramatically to the physician with nasal obstruction, epistaxis, visual loss, anosmia and proptosis.

Surgery is usually the primary treatment of choice followed by radiation therapy in malignant lesions. With current imaging technology, we can accurately define the extent of lesion and determine which structures are involved. The subcranial lesions can be dealt with transfacial approaches, such as lateral rhinotomy and midfacial degloving. In the past two decades, endonasal endoscopic techniques have advanced sufficiently and proven outcomes in world literature to establish it as another approach to sinonasal tumors.

With a constant endeavor to reduce morbidity and improve quality of life of the patient, the endonasal access has progressed in leaps and bounds. Today, the expanded endonasal approach in a modular fashion as described and popularized by Professor A min Kasam, Professor Ricardo Carrau and Professor Carl Synderman from the University of Pittsburgh has been accepted world over. Transcranial lesions of the anterior skull base can today be resected using the nasal corridor with oncologic safety. This approach is minimal in access but maximum in intent.

\section{Traditional Craniofacial Resection}

AS Ketcham ${ }^{1}$ in the seventies popularized the combined approach to anterior skull base lesions which reached or crossed the skull base. These lesions would involve the ethmoids and the cribriform plate. Tumors could be cleared from 'below' using the transfacial access via medial maxillectomy or total maxillectomy and cleared from 'above' via the transfrontal craniotomy. The ethmoid and maxillary sinuses could be cleared completely and the dura and involved brain at times could be resected for an oncologically sound clearance. A s borne out by the study by I nternational Collaborative Study Group ${ }^{2}$ formed by 17 institutions from around the world, the overall survival at 5 years was $54 \%$ with a median follow-up of 25 months. The higher grade tumors, those with intracranial extent and those with positive margins did uniformly poorly. A Il data concerning malignant tumors will be compared with this large study of 1,307 patients.

Transnasal endoscopic tumor resection results have been published from major centers around the world. Starting from the early nineties groups in B el gium, A ustria, Germany and Italy have been steadily removing select tumors with improving results. Stammberger $\mathrm{H}_{\text {et }} \mathrm{l}^{3}$ from $\mathrm{G}$ raz, A ustria published encouraging report of 36 malignant paranasal sinus tumors resected endoscopic with the esthesioneuroblastomas having 100\% survival with 37 months follow-up. Goffart $Y$ et al ${ }^{4}$ from Belgium published a retrospective study of 78 cases from two centers in B el gium. They reported 2- and 5-year survival rates of 73.1 and $52.3 \%$ respectively among the entire group, while the adeno- 
carcinoma group exhibited a significantly better prognosis than other histological types with 2- and 5-year survival rates of 89.8 and $63.8 \%$ respectively. Recently, Nicolai $P$, Capabianca $P$ et al $^{5}$ from two centers in Italy reported on a 10-year data with 184 malignant tumors. The most frequent histotypes encountered were adenocarcinoma (37\%), squamous cell carcinoma (13.6\%), olfactory neuroblastoma $(12 \%)$, mucosal melanoma $(9.2 \%)$ and adenoid cystic carcinoma (7.1\%) and they reported a 5-year diseasespecific survival of $91.4 \%$ for those resected exclusively endoscopically and $58.8 \%$ for those resected with cranioendoscopic techniques. From USA Hanna ${ }^{6}$ et al of the M D A nderson cancer center reported 120 cases treated endoscopically out of which 27 cases were treated with endoscopic-assisted CFR and the 5 years disease-specific survival were $87 \%$ with a medial follow-up of 37 months. So, there is enough evidence that endoscopic resection is oncologic and safe, if you adhere to oncologic principles.

\section{Concept}

\section{Why Endonasal Approach?}

Today, we have a better understanding of endoscopic skull base anatomy, improved technology and a better understanding of the disease biology. Disease control via complete tumor resection has remained the objective. But, there has been a paradigm shift in achieving it. The en bloc resection method is gold standard and there is no disputing its efficacy. But, truly the actual ability to achieve en bloc resection in these tumors is in doubt. These tumors seldom come out intact and the posterior regions of these tumors are al ways removed piece meal. H ence, this led to a rethink on the exact end point of the surgery which is a completely resected base of the tumor with margins. Whether you can achieve this via an en bloc surgery or via a piece meal resection is immaterial so long as the tumor has an oncologically safe clearance. This same logic has been proven in transoral laser microsurgery be oncologically safe. Thus, if there is a planned surgical method detailing exact structures to be removed to achieve a complete, oncologically safe tumor resection then endonasal endoscopic resection, if feasible should be as safe as conventional surgery.

Sinonasal tumors lend themselves for endoscopic resection. These tumors arise in and around the ethmoids, growing locally within the nasal cavity and extend laterally, superiorly and posteriorly. These tumors tend to hang into the nasal cavity rather than infiltrate the surrounding structures. A diagnostic nasal endoscopy (DNE) usually reveals these tumors not involving the very structures they are reported to be involved on imaging. DNE should be a part of a routine evaluation, providing us three-dimensional understanding of the tumor spread which in addition to the imaging will help us to plan the surgical options.

In traditional surgery, the resection is standard irrespective of involvement of the structures. Medial maxillectomy involves removal of the frontal process of the maxilla, lacrimal bone, lamina papyracea part of maxilla medial to the infraorbital foramen, the middle turbinate and the inferior turbinate along with the lacrimal sac and the nasolacrimal duct. Thus, even though the nasolacrimal duct system is uninvolved, in a conventional medial maxillectomy we end up with marsupialization of the lacrimal sac and a potential for epiphora.

In oncologic surgery, margins are essential for ensuring a safe resection. H ow ever, when we consider margins during extirpation of sinonasal and anterior skull base tumors, we are limited by the orbit laterally, the brain superiorly, the optic nerves and internal carotid artery posterolaterally and the pituitary gland posteriorly. Conventional surgical margins as in case of other tumors would entail removal of adjacent vital structures which would significantly increase morbidity and decrease quality of life. When we deal with tumors extending to the sphenoid, the margins are even narrower. When the tumor extends intracranially, the superior margin would be the dura which can be resected in a limited way till we reach the optic chiasma posteriorly. It is not possible to remove more basal dura. $M$ argins in these resections are still possible. To get a complete tumor clearance in this confined space, we need to enter the adjacent compartment. In order to be sure of a complete clearance of ethmoids, we need to remove the lamina papyracea and expose the periorbita.

Today, medial maxillectomy, ethmoidectomy, frontoethmoidectomy, sphenoidectomy and anterior craniofacial resection all are feasible endonasally. What the endoscopic approach offers is ability to remove the tumor under magnification and better illumination. It allows us to tailor the resection to the tumor with the objective of removing the tumor base completely with margins as much as possible in that region preserving uninvolved structures.

\section{Case Selection (Fig. 1)}

The sinonasal and anterior skull base tumors require a threedimensional resection. The sinonasal tumor should be feasible for complete excision via traditional methods and in addition there should not be involvement of the anterior maxillary wall, outer table of the frontal bone and skin. The transcranial component should not be extensive with dural enhancement extending laterally. The optic nerve, optic chiasma, internal carotid arteries and the optic apex should be free of tumor. Subcranial involvement of the pterygopalatine fossa and the internal carotid artery are contraindications to surgery. 

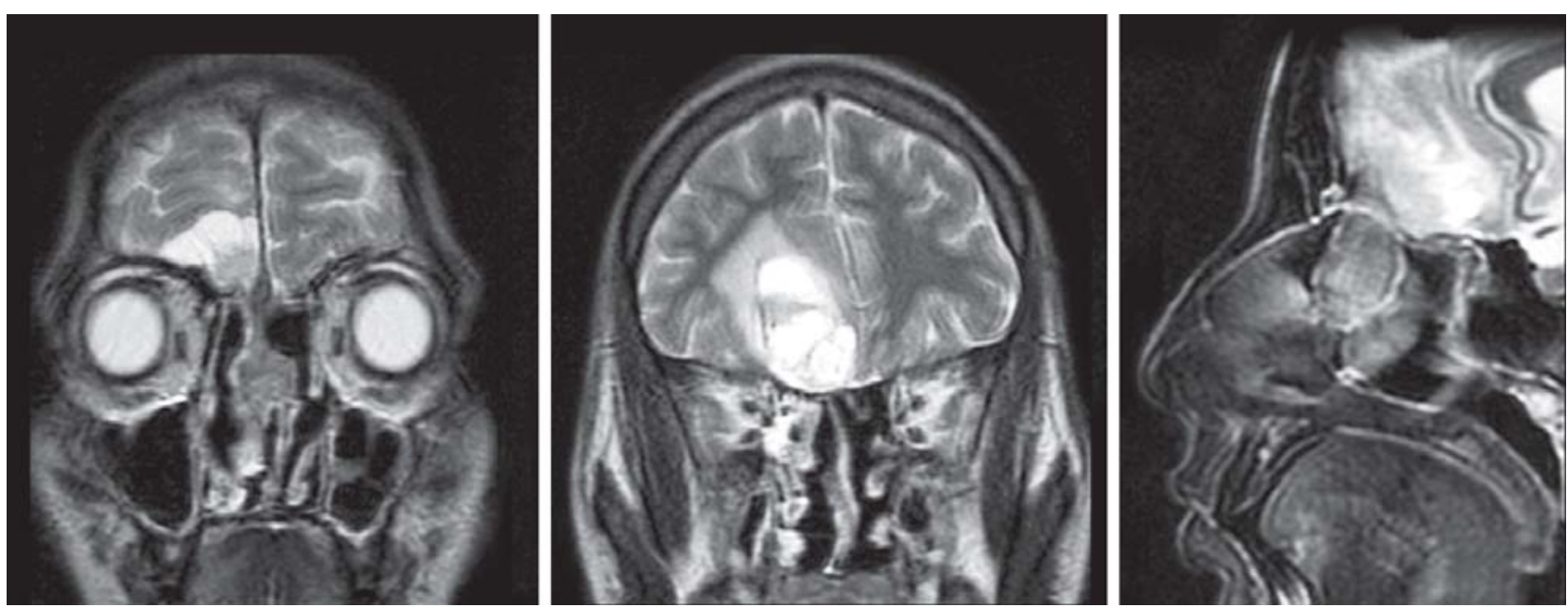

Fig. 1: MR showing limited nasal tumor with transcranial spread

\section{Steps}

\section{Endonasal Endoscopic Access}

Endoscopic survey of the lesion in the nasal cavity is carried out following which the tumor is central debulked allowing the lining of the tumor to fall on itself. Thereafter a complete clearance of the tumor is attempted entering adjacent compartments for margins. The frontal sinus is exposed and communicated with opposite frontal sinus (Endoscopic Draf III procedure). The maxillary sinuses are exposed. The sphenoid is opened exposing the planum followed by removal of the septum. The exposure of the skull base from frontal sinus to the planum sphenoidale is completed. The ethmoid arteries are then cauterized either unilaterally or bilaterally as needed. The endonasal access is completed exposing the tumor at the skull base.

\section{Transcranial Access (Fig. 2)}

Bicoronal incision is taken followed by elevation of the scalp anteriorly and posteriorly above the galea aponeurotica identifying the supratrochlear vessels anteriorly. A galealpericranial flap is prepared at this point taking adequate length for a proper skull base reconstruction. The frontal craniotomy is planned using as few burr holes as possible. The dura is exposed and tumor identified by retraction of the frontal lobe. The cribriform plate is exposed after dividing the olfactory bulbs.

The tumor is then circumscribed removing the involved structures including dura and involved brain, if present. The cribriform plate region is then prepared for osteotomy cuts transcranially. While the cuts are being placed, the endonasal skull base is kept under vision to avoid trauma to the eye, etc. The tumor along with the skull base is delivered transcranially. This completed the surgical removal.

\section{Skull Base Repair}

Dural repair is carried out with fascia lata. The skull base repair is done with the galeal-pericranial flap which is anchored to bone near the planum sphenoidale. After replacing the calvarium, it is secured with either plates or suture. The overlying scalp is closed over a suction drain. $\mathrm{N}$ asal packs are inserted which are preferably polyvinyl alcohol sponges.

\section{Postoperative Period}

Complete bed rest is preferred with head elevation. Intravenous antibiotics are given for 5 days. Patient is advised not to suppress a sneeze and to open his mouth during a sneeze. Stool softners are prescribed to avoid straining. $\mathrm{N}$ asal packs are removed on fifth day.

\section{Advantages over Traditional Craniofacial Resection (Fig. 3)}

The transcranial access is standard with all its antecedent issues. W ith an endonasal access, we can avoid the facial incision besides preserving uninvolved structures, such as the frontal process of maxilla, lacrimal bone, nasolacrimal sac and duct. Disassembly of facial bones is circumvented and, in the long run, the possibility of bone resorption leading to facial disfigurement is avoided. During tumor resection, we are directly on the tumor without having to go through skin and bone removal. Hence, the endoscopic access is rapid, safe and cosmetic. The endoscopic-assisted craniofacial combines cosmetic and oncologically sound transnasal access with the safety of transcranial access.

\section{RESULTS}

From September 2002 to December 2011, we have performed 137 endonasal resections of benign (26) and 


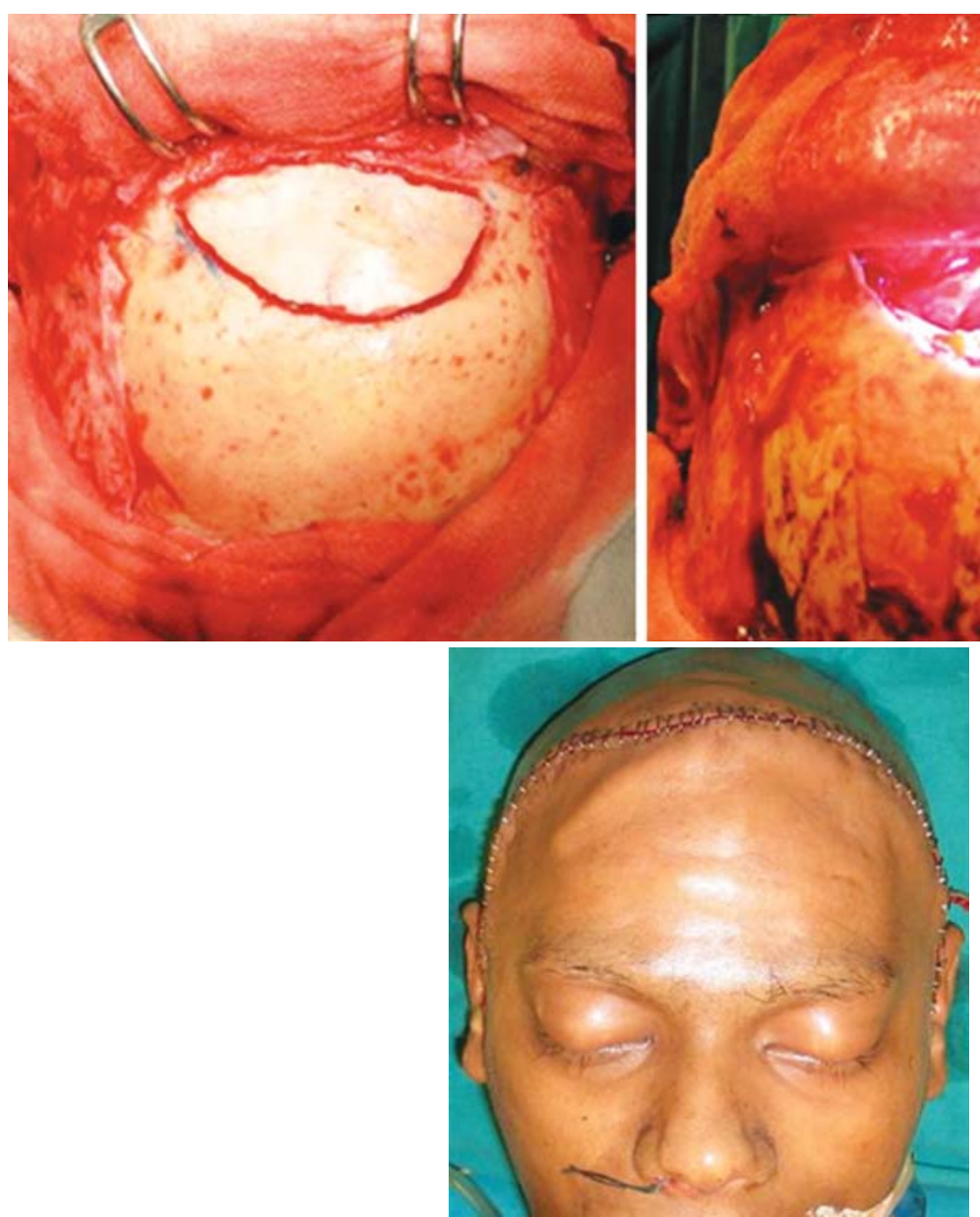

Fig. 2: Transcranial access via bicoronal flap and craniotomy and transnasal clearance
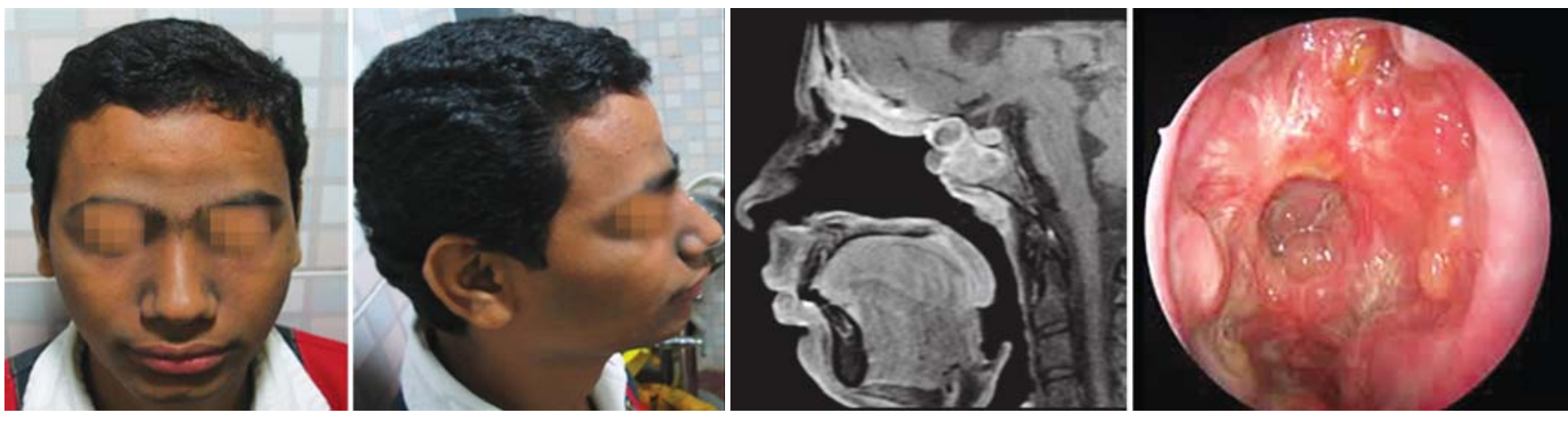

Fig. 3: Postoperative results showing excellent cosmesis, good cranionasal separation and well-mucosalized nasal cavity

malignant (111) lesions. Endonasal endscopic resection was performed in 120 patients of which two needed to be converted to open procedures, one for control of hemorrhage whilst the other was for oncologic safety. Endoscopic-assisted craniofacial resection was performed in 12 patients, while complete transnasal cranioendoscopic resection was performed in five patients. A mong the 111 malignant lesions, most common were adenocarcinomas (18\%) followed by esthesioneuroblastomas 13\% and adenoid cystic carcinoma $9.5 \%$ among other varied histologies. Complications seen were suspect CSF leak in two patients. In one patient, the skull base was repacked, while in the other, the patient recovered without any intervention. In both cases, there was no conclusive evidence of leak. L ongterm results will be published subsequently.

\section{CONCLUSION}

M inimal access surgery for sinonasal and anterior skull base tumors is now an established modality with proven safety. The endonasal access should be used whenever, feasible alone and in combination with external approaches with the aim of not only an optimal resection but also a better quality of life as well. 


\section{REFERENCES}

1. Ketcham AS, Wilkins RH, V an Buren JM. A combined intracranial facial approach to the paranasal sinuses. A m J Surg 1963;106:698-703.

2. Patel SG, Singh B, Polluri A, et al. Craniofacial surgery for malignant skull base tumours. Report of an International Collaborative Study. Cancer 2003;98:1179-87.

3. Stammberger $\mathrm{H}, \mathrm{A}$ nderhuber $\mathrm{W}, \mathrm{W}$ alch $\mathrm{C}$, Papaefthymiou G. Possibilities and limitations of endoscopic management of nasal and paranasal sinus malignancies. A cta Otorhinolaryngol Belg 1999;53(3):199-205.
4. Goffart $Y$, J orissen $M$, Daele J, et al. M inimally invasive endoscopic management of malignant sinonasal tumours. A cta Otorhinolaryngol B elg 2000;54(2):221-32.

5. Nicolai $P, B$ attaglia $P, B$ ignami $M$, et al. Endoscopic surgery for malignant tumors of the sinonasal tract and adjacent skull base: A 10-year experience. Am J Rhinol M ay-Jun 2008;22(3): 308-16.

6. Hanna E, DeM onte F, I brahim S, et al. Endoscopic resection of sinonasal cancers with and without craniotomy: Oncologic results. A rch Otolaryngol Head Neck Surg Dec 2009; 135(12):1219-24. 